\title{
Visita domiciliaria y participación de las familias en la educación
}

Home Visit and Family Participation in the Education

Artículo de investigación | Research Article

Fecha de recepción: 02 de agosto de 2018

Fecha de aceptación: 12 de abril de 2019

Fecha de disponibilidad en línea: marzo de 2020

doi: 10.11144/Javeriana.m13.vdpf

\section{Alicia Cristina Razeto-Pavez} arazeto@uc.cl

Pontificia Universidad Católica de Chile, Chile D) ORCID: https://orcid.org/0000-0003-2887-8424

Para citar este artículo | To cite this article Razeto-Pavez, A. C. (2020). Visita domiciliaria y participación de las familias en la educación. magis, Revista Internacional de Investigación en Educación, 13, 1-25. doi: 10.11144/Javeriana.m13.vdpf 


\title{
Resumen
}

Este artículo explora el proceso de visitas domiciliarias hechas a familias por parte de trabajadores sociales ubicados en las escuelas. La metodología fue cualitativa, e implicó hacer entrevistas a los trabajadores sociales y la observación de campo durante el proceso de visitas domiciliarias. Los resultados muestran que las visitas domiciliarias se utilizan principalmente en casos de ausentismo escolar y con el propósito de obtener información. De este modo, las familias se sienten más valoradas por la escuela.

\section{Palabras clave}

Hogar; participación de los padres; servicio social; trabajo social

\begin{abstract}
This study aims to explore the process of home visit to the family by the social workers belonging to the schools. The methodology was qualitative and included interviews to social workers and observations out in the field during the home visit process. The results show that the home visits are used mainly in cases of student absences and are intended to gather information. This way the families feel more appreciated by the school.
\end{abstract}

\section{Keywords}

Parent participation; houses; social work; social services 
Descripción del artículo | Article description

En este artículo de investigación se muestran los resultados del estudio La visita domiciliaria como estrategia de intervención social para aumentar la participación de los padres en la educación de sus hijos en escuelas básicas municipales. Proyecto Fondecyt 11140679 de Conicyt-Chile.

\section{Introducción}

La participación que tienen las familias en la educación de los niños es fundamental para potenciar sus capacidades e incidir en sus resultados académicos (Baquedano-López, Alexander \& Hernández, 2013; Boberiene, 2013; Dikkers, 2013; Garcia-Reid, Peterson \& Reid, 2013; Vera, González \& Hernández, 2014). El espacio de aprendizaje por excelencia es el hogar, seguido del barrio, la comuna y la ciudad (Blanco \& Umayahara, 2004). En este continuo, la escuela viene a "fortalecer con su conocimiento especializado lo que la familia ha iniciado y continúa realizando" (Blanco \& Umayahara, 2004, p. 23)

Si se considera la vital importancia que poseen las familias en la educación, entonces es primordial poner en marcha estrategias para fortalecer las capacidades de los padres y las escuelas para una efectiva participación ${ }^{1}$ (Hoover-Dempsey et al., 2006). Es fundamental reforzar la alianza con las familias desde las instituciones escolares para atender a todos los estudiantes, en especial a los que proceden de contextos socialmente desfavorecidos (Gil, 2009). Por lo tanto, es necesario que las escuelas busquen nuevas formas de abordaje y trabajo interdisciplinario que permitan la colaboración con los padres - considerándolos como agentes de cambio- a través de apoyo recíproco, información y asesoría de la escuela cuando sea necesario (Gil, 2009).

En sintonía con ese cometido, Razeto (2016b) propone a la visita domiciliaria como una estrategia de alto potencial que puede poner en marcha la escuela para incentivar la participación de los padres menos involucrados en la educación de sus hijos y mejorar su vinculación con la escuela. Si bien la realización de visitas domiciliarias desde los centros educativos no está directamente contemplada por la legislación chilena ni por la política educacional, es una práctica que tiene una larga tradición en Chile. Las primeras evidencias de la realización de visitas domiciliarias en el contexto escolar

1 En este artículo se utilizarán indistintamente los términos participación e implicación como traducción de involvement. 
datan de la década de los años 30 del siglo XX, luego de la fundación de las primeras escuelas superiores de servicio social, formadoras de las primeras visitadoras sociales de grado profesional² (Zamorano, 1935).

No solo en Chile existen experiencias sobre visitas domiciliarias en el contexto educacional. También se aplica este tipo de práctica en Brasil ${ }^{3}$ como estrategia de aproximación de los agentes de la escuela hacia las familias (Castro \& Regattieri, 2012). En este país, en algunos casos las visitas las realizan los profesores, en otros, psicólogos o asistentes sociales. En ocasiones, las hacen jóvenes universitarios o que han concluido su enseñanza media, quienes son seleccionados y formados para tal fin. También hay prácticas de visitas domiciliarias en varios estados de los Estados Unidos, como las documentadas a través de la experiencia Parent Teacher Home Visits (PTHV), como estrategia para comprometer a las familias, los educadores y los estudiantes como equipo en el proceso educativo (Sheldon \& Jung, 2018).

Por el lado de la investigación, hay estudios que plantean que las visitas pueden promover una implicación familiar positiva en la educación (Allen \& Tracy, 2004; Franklin \& Lagana-Riordan, 2009), y reconocen múltiples motivos por los que estas pueden llevarse a cabo, como la inasistencia a la escuela, el bajo desempeño académico, el embarazo adolescente, los problemas en la alfabetización, los problemas de salud mental, el abuso de drogas, la integración de estudiantes migrantes y los problemas económicos (Franklin \& Lagana-Riordan, 2009; Paulsell, Boller, Hallgren \& Mraz, 2010).

Considerando estas ventajas, el presente estudio explora los usos que actualmente se le dan a la visita domiciliaria en Chile en el contexto escolar, describiendo sus posibilidades como estrategia para avanzar hacia el encuentro entre las familias y escuelas, con miras a apoyar el aprendizaje de los estudiantes. Este encuentro no solo se desarrolla en los tiempos y espacios institucionales escolares, sino también en los extraescolares o en los distintos momentos de la vida cotidiana familiar en el hogar, pues el hogar del estudiante, su ambiente más próximo, también puede ser un espacio crucial de encuentro entre la escuela y la familia.

En Chile, las visitas domiciliarias en el contexto escolar han sido principalmente desarrolladas por trabajadores sociales que se desempeñan laboralmente en centros educativos de enseñanza primaria o secundaria. A partir del año 2008, comenzó a incrementarse la presencia de trabajadores

2 En Chile, a partir de la década de los años 50, el título profesional era de asistente social. En la actualidad, es de trabajador social.

3 En Taboão da Serra (estado de San Pablo), los profesores realizan visitas domiciliarias a todos sus alumnos como forma de interactuar con las familias e involucrarlas en el proceso educativo. 
sociales en los centros educativos, debido a que estos empezaron a recibir nuevos recursos en el marco de la Ley de Subvención Escolar Preferencial, la cual reconoce que se requieren recursos extraordinarios para satisfacer las necesidades educativas de los niños y las niñas (Arevalo \& Santana, 2015). La principal labor de los trabajadores sociales de escuelas es ser un puente entre la escuela y la comunidad, proveyendo servicios a los estudiantes, las familias y el staff para promover y apoyar el éxito académico y social de los estudiantes (School Social Work Association of America, 2019). "En el mundo angloparlante, se denomina Trabajo Social Escolar a la subdisciplina que se encarga de la intervención social en las escuelas" (Villalobos, 2015, p. 57). De acuerdo con Villalobos (2015), los trabajadores sociales de las escuelas pueden generar procesos de apoyo a los estudiantes en su aprendizaje, potenciando la cimentación de entornos académicos positivos y equitativos, promoviendo el desarrollo de apoyos sociales, psicológicos, biológicos y mentales de los estudiantes y sus familias.

\section{Conceptualización}

La visita domiciliaria ha sido utilizada por educadores, médicos, enfermeras y trabajadores sociales como una herramienta efectiva para proveer apoyo y servicios a los niños y sus familias (Cook \& Sparks, 2008; Wasik \& Bryant, 2001). En su nivel más básico, según Wasik \& Bryant (2001), la visita domiciliaria podría definirse como una estrategia que contempla un proceso de ayuda a una familia por parte de un agente visitador, el cual se desarrolla en el propio hogar y durante un período de tiempo extenso. La ayuda o el servicio de información u orientación entregado a las familias pueden enfocarse en cubrir diversas necesidades de carácter social, emocional, cognitivas, de salud o educativas (Wasik \& Bryant, 2001).

La visita domiciliaria puede dirigirse a una persona o a una familia, abarcando desde la atención prenatal hasta la ancianidad (Aracena et al., 2011). En todos los casos, la principal característica es que se aplica en el propio hogar y a través de encuentros habituales a lo largo del tiempo, por lo que es una herramienta óptima para poblaciones en condiciones de pobreza o exclusión social, por sus dificultades para acceder o desplazarse hacia los sistemas formales de atención social (Aracena et al., 2011). En este sentido, las visitas domiciliarias contribuyen a superar barreras tales como enfermedad, discapacidad física o presencia de necesidades especiales, falta de transporte, falta de cuidado de niños, baja motivación y alienación desde el sistema educacional o de salud (Wasik \& Bryant, 2001).

Las visitas domiciliarias ofrecen la oportunidad única de obtener información relevante acerca del ambiente familiar, sus recursos y necesidades, 
además de la oportunidad de prestar un servicio individualizado. Adicionalmente, transmiten un mensaje de respeto y aprecio por las necesidades de las familias (Wasik \& Bryant, 2001).

El bienestar y el desarrollo social de los niños es uno de los principales propósitos de la visita domiciliaria (Wasik \& Bryant, 2001), por lo cual, el sistema escolar se presenta como un contexto idóneo para aplicarlas, pues los niños permanecen en él durante muchos años y varias horas al día. Según Allen \& Tracy (2004), las visitas domiciliarias pueden mejorar la conexión entre la escuela y la familia, bajo el entendido de que los padres no necesariamente tienen que ingresar a la escuela para influir e implicarse en la educación de sus niños, pues hay actividades importantes que pueden desarrollarse desde el hogar. En su reconocido trabajo sobre el tema de la alianza familia-escuela, Epstein (2011) identifica a la visita domiciliaria como una de las técnicas disponibles para fomentar la participación de los padres, entendiendo que el contacto y las relaciones personales entre estos y los profesores son vitales para establecer un compromiso por parte de los padres para que continúen reforzando el aprendizaje en el hogar.

El potencial de aplicarlas desde el ámbito escolar radica en que la visita es "una estrategia que puede facilitar la participación de los padres en la educación de sus hijos y ayudar a entender mejor el contexto extraescolar y sus influencias en el aprendizaje, cuestiones claves para las escuelas básicas" (Allen \& Tracy, 2004, p. 7). Entre las ventajas que tienen, se puede mencionar que las visitas pueden ayudar a resolver las barreras a la participación de los padres en la educación de sus hijos, como la falta de tiempo para ir a reuniones o para leer materiales enviados a la casa, no sentirse valorado ni bienvenido en la escuela, no comprender el sistema escolar ni su lenguaje, y la accesibilidad (Allen \& Tracy, 2004). Por estas razones, las visitas domiciliarias proveen mejores mecanismos para responder a la diversidad cultural de las familias de los estudiantes, bajo el supuesto de que cada cultura puede lograr el involucramiento de maneras distintas o a través de distintos caminos, los que pueden ser infravalorados o malinterpretados por la escuela. De este modo, las visitas contribuyen a que el personal de la escuela aprenda acerca de la diversidad de las familias y cómo comunicarse con ellas.

Por otra parte, el hecho de que la provisión de los servicios se realice en su ambiente natural "facilita la detección y comprensión de las necesidades familiares junto con la individualización de los servicios para responder a ellas" (Allen \& Tracy, 2004, p. 198). Cabe señalar que el ambiente familiar contribuye a equilibrar la relación asimétrica de poder entre el profesional y la familia, lo que ayuda a construir relaciones de confianza (Allen \& Tracy, 2004). Sin embargo, este equilibrio no es automático, y solo se logrará si la persona que realiza la visita domiciliaria busca aliarse con la familia y tiene 
una actitud de respeto hacia ella, de manera que se desarrolle una relación de confianza que ayude a equiparar el poder entre la familia y la escuela (Cook \& Sparks, 2008).

Es así como el agente visitador tiene que desarrollar ciertas habilidades y contar con capacitación y conocimientos técnicos para mostrarse competentes en la entrega del servicio (Aracena et al., 2011; Avellar \& Supplee, 2013; Tubach et al., 2012; Wasik \& Bryant, 2001), logrando una relación cercana y de alianza con la familia, que ayude a aumentar su compromiso con la educación del niño.

Entre otras de sus ventajas también se encuentra el potencial impacto a nivel pedagógico, puesto que,

cuando la escuela mejora su conocimiento y comprensión de los alumnos, crece su capacidad de comunicación y adecuación de las estrategias didácticas, con el consiguiente aumento de las oportunidades de realizar un trabajo escolar exitoso. En este sentido, la conquista de la tan deseada participación de las familias en la vida escolar de los alumnos debe ser vista como una parte constitutiva de la planificación educativa (Castro \& Regattieri, 2012, p. 7).

Asimismo, Castro \& Regattieri (2012) postulan que, cuando los estudiantes perciben que sus profesores conocen la situación familiar, tienen más seguridad para expresar sus dudas y temores en el aula. Al mismo tiempo, estas autoras señalan que, al conocer la situación de los estudiantes, los profesores comprenden mejor su comportamiento y tienen unas expectativas más realistas. De este modo, es fundamental que los trabajadores sociales que realizan las visitas compartan la información socioeducativa de las familias con los profesores, si es que estos no se implican en la realización de las visitas y si se cuenta con el consentimiento de la familia. Cabe señalar que existen experiencias de intervención en las que los profesores realizan visitas domiciliarias en el contexto educacional, como el caso del Family Engagement Partnership. Una evaluación a este programa por parte del Center for Social Organization of Schools, en la Universidad Johns Hopkins (Center for Social Organization of Schools, John Hopkins University, 2015), mostró que la visita domiciliaria de los profesores a sus estudiantes de bajos ingresos mejoraba su asistencia y su comprensión lectora.

\section{Metodología del estudio}

El estudio tuvo por objetivo describir cómo, para qué y con qué frecuencia realizan las visitas domiciliarias los trabajadores sociales que se desempeñan en establecimientos educativos primarios y públicos. Este estudio 
se realizó con un enfoque metodológico cualitativo, con el fin de comprender en su contexto cotidiano la forma en la cual las visitas domiciliarias son definidas, valoradas y puestas en marcha por los trabajadores sociales participantes del estudio. Considerando el escaso estudio acumulado en el tema desde el sistema educativo, se optó por un tipo de estudio de nivel exploratorio, que contribuya a iniciar un proceso de familiarización con esta estrategia implementada en las escuelas chilenas, para avanzar hacia futuros estudios sobre su efectividad.

La muestra estuvo conformada por 10 profesionales: 9 trabajadores sociales y 1 psicólogo, ${ }^{4}$ todos ellos con contrato laboral vigente en distintas escuelas municipales básicas de la Región Metropolitana de la ciudad de Santiago, Chile.

Estas escuelas tenían la característica común de poseer un alumnado con familias de ingresos bajos o medios-bajos. Algunos de estos centros educativos estaban emplazados en sectores periféricos de la capital y otros, en sectores más centrales, pero con un alumnado proveniente del extranjero, inmigrante o de un estrato socioeconómico medio-bajo.

Los participantes fueron seleccionados de manera intencionada para lograr una comprensión más profunda de las visitas domiciliarias en el contexto escolar. El disponer de una relación previa con las instituciones educativas fue un criterio importante, pues facilitó la viabilidad del trabajo de campo. Junto a este, otro criterio fue el de la voluntariedad de los individuos para participar en el estudio.

Se contemplaron dos técnicas principales como estrategias de recolección de información. Primero, la entrevista a cada profesional participante $y$, segundo, la observación de tres sesiones de visitas domiciliarias realizadas por los profesionales a distintas familias de los estudiantes de los establecimientos educativos. El orden de aplicación de estas técnicas fue: en primer lugar, la entrevista semiestructurada, con el fin de conocer al participante, establecer un vínculo, captar sus significaciones y valoraciones acerca de las visitas domiciliarias, conocer en su discurso sus formas de aplicación y coordinar el proceso de observación a las visitas que el profesional realizaría. En segundo lugar, se hizo la observación de las visitas domiciliarias, con el objetivo de conocer in situ el contexto de aplicación de la estrategia, los objetivos, la forma en la que esta era implementada y sus dificultades

4 De los 10 profesionales participantes del estudio, 2 de ellos formaban una dupla de trabajo en una misma escuela ( 1 trabajador social y 1 psicólogo). A ellos se les realizó una entrevista grupal y se les observó en conjunto. Todos los demás profesionales (8) trabajaban individualmente en escuelas distintas. 
y oportunidades. Debe señalarse que la coordinación y gestión de las visitas fue realizada por el profesional de la escuela, como parte de su labor habitual. El papel del investigador durante la visita solo se limitó a la observación, sin intervenir verbalmente durante el proceso de interacción entre el profesional y la familia. Además, se realizó el registro de la visita una vez que esta se daba por finalizada, para no sesgar el contexto de observación. Por esta misma razón, no se hizo grabación visual ni auditiva de las visitas, pues el propósito era no interferir ni modificar la interacción natural que se produce entre el profesional y los miembros de la familia. Así, se buscó evitar conductas de inhibición o comportamientos artificiales que pudieran producirse por parte de los miembros de la familia, al estar poco habituados a situaciones de investigación social.

Es preciso mencionar que, durante las visitas, el investigador tomó notas generales. Una vez concluidas, se dirigía a un parque cercano o sitio tranquilo del barrio para realizar el registro completo y cubrir el formulario de la pauta de observación. En promedio, este proceso duraba unos 30 minutos. Debe señalarse que los investigadores estaban entrenados en el contenido de la pauta de observación, y mencionar que estos eran profesionales con experiencia en otras investigaciones sociales y en el uso del método cualitativo.

Desde el punto de vista ético, es preciso destacar que cada uno de los profesionales firmó un consentimiento informado, en el cual se explicaba el propósito del estudio, las ventajas y riesgos de participar en este y las garantías de anonimato y confidencialidad, para lo que se incluyó un formulario para su firma libre y voluntaria de aceptación de la participación. Este consentimiento abarcaba tanto la participación de los profesionales en las entrevistas como en las observaciones de campo. Consecuentemente con el objetivo del estudio, el foco principal de la observación estuvo puesto en el desenvolvimiento de la intervención del trabajador social durante las visitas y no en el desarrollo del caso social o familiar. De igual forma, en el momento de realizar la visita domiciliaria, el integrante de la familia que atendió a los profesionales firmaba voluntariamente un consentimiento de aceptación de la presencia del investigador.

Las entrevistas fueron realizadas en las oficinas de las profesionales ubicadas en las escuelas; estas fueron grabadas, y en promedio tuvieron una duración de 30 minutos. Se obtuvo un total de 9 entrevistas. Una vez efectuadas, se dio paso a la coordinación de las observaciones de las visitas domiciliarias. Se lograron 27 observaciones, a partir de 27 visitas domiciliarias efectivas. Con respecto a estas, es preciso mencionar algunos aspectos relevantes desde el punto de vista investigativo: 
- Se realizaron en el transcurso de un período de cinco meses, entre mayo y septiembre del año 2016.

- Fueron elegidas por los profesionales de las escuelas, sin presiones ni sugerencias.

- Fueron coordinadas a través de correo electrónico o contacto telefónico.

- El punto de encuentro entre investigadores y profesionales fue la escuela, y la forma de desplazamiento fue caminando o en taxi.

El equipo de investigadores que hizo las entrevistas y observaciones estuvo compuesto por tres personas: el investigador responsable y dos miembros del equipo de investigación. Asimismo, este investigador responsable y uno de esos integrantes del equipo participaron del proceso de codificación de las entrevistas y observaciones. Estos, inicialmente, realizaron la codificación de forma independiente y, posteriormente, pusieron en común lo trabajado, estableciendo diferencias y similitudes.

Tanto las entrevistas como las observaciones fueron efectuadas de acuerdo con un instrumento previamente confeccionado por el equipo de investigación. El instrumento de la entrevista consistió en una pauta estructurada y ordenada de preguntas abiertas, con las siguientes categorías:

1. Situaciones o motivos que dan origen a la visita domiciliaria

2. Propósito de la visita domiciliaria

3. Frecuencia en la realización de visitas domiciliarias

4. Actores de la visita: agentes y personas visitadas

5. Aviso de la visita domiciliaria y respuesta de la familia

6. Percepción de efectividad de la visita domiciliaria

7. Dificultades y ventajas de la visita domiciliaria

Por su parte, la observación se realizó con la guía de una pauta de registro estructurada, orientada a captar la presencia o ausencia de ciertas acciones o conductas del agente o del usuario durante las distintas etapas de las visitas. Esta pauta, además, permitió registrar de manera abierta las dificultades o facilitadores de cada etapa. El instrumento contempló las siguientes dimensiones y subdimensiones de observación:

1. Coordinación y planificación de la visita domiciliaria: por qué se realiza, coordinación con padres, información a los padres, plan de visita, forma de traslado al hogar, identificación del agente visitador.

2. Inicio de la visita domiciliaria en el hogar: saludo, acogida, introducción, habitación, distractores. 
3. Desarrollo de la visita domiciliaria: pauta de preguntas, padres responden, registro, emociones y consejería.

4. Finalización de la visita domiciliaria: conclusión, compromisos, agradecimiento, despedida.

5. Posrealización de la visita domiciliaria: uso del registro, reporte a superiores.

La estrategia de análisis de la información cualitativa consistió en un análisis temático, siguiendo a Braun \& Clarke (2006), desarrollando una aproximación primordialmente deductiva a los temas de análisis. Es preciso señalar que el nivel de análisis fue principalmente semántico y no latente, de acuerdo con el objetivo del estudio.

Por otra parte, se realizó un análisis cuantitativo descriptivo básico de la información recolectada en las 27 observaciones, la que fue sistematizada y tabulada en un archivo Excel de Microsoft Office.

Con el propósito de lograr una presentación integrada de los resultados del estudio, se optó por organizarlos de acuerdo con las categorías más relevantes, incluyendo la información de las entrevistas y las observaciones. Estas categorías fueron las siguientes: 1) problemáticas socioeducativas que originan la realización de las visitas domiciliarias; 2) propósito de la visita domiciliaria; 3) actores de la visita domiciliaria: agente visitador y persona visitada; 4) aspectos éticos de la visita domiciliara: presentación, información, coordinación de la visita; 5) percepción de la efectividad de la visita domiciliaria, y 6) dificultades y ventajas de la visita domiciliaria en el contexto escolar.

\section{Resultados de la investigación}

\section{Problemáticas socioeducativas que originan}

\section{la realización de las visitas domiciliarias}

Una de las principales situaciones socioeducativas que motivan la realización de las visitas domiciliarias es el ausentismo escolar, con un 64\% de las visitas observadas. Esta cifra está muy por encima de otras situaciones, tales como problemas familiares de violencia u otros (14\%), problemas socioeconómicos (11\%) o de disciplina en la sala de clases (11\%).

La información arrojada por las observaciones concuerda con la obtenida en las entrevistas, en las que se destaca el fenómeno de la inasistencia como la principal razón que da origen a la actuación profesional a través de la visita, tal como se aprecia en el discurso del entrevistado:

Tiene que ver con la inasistencia de los niños a clases. Cuando los niños faltan reiterativas veces hay todo un protocolo: primero, los profesores tienen que generar acciones; si esas acciones no se cumplen pasa a 
inspectoría general; inspectoría general cita al apoderado, y ya cuando los compromisos no son cumplidos pasan a la asistencia social, y yo voy a hacer visita (E1).

En el ámbito de las estrategias de actuación frente al ausentismo, hay varios autores que ven la visita domiciliaria como una estrategia efectiva para disminuir las tasas de ausentismo de los estudiantes, ayudando en caso de familias pasivas, aisladas o que tienen menos motivación para asistir a la escuela (Kearnet, 2016; Kim \& Streeter, 2013; Reid, 2013). No obstante, también es preciso mencionar que la visita domiciliaria no es la única estrategia de intervención frente a este problema, puesto que también existen los enfoques grupales de intervención, de entrenamiento en habilidades sociales a estudiantes y familias (Lauchlan, 2003; Pellegrini, 2007), los cuales no son mencionados por los entrevistados.

Otro de los motivos que las originan se relacionan con situaciones de vulneración de derechos y problemas familiares, tales como la violencia intrafamiliar, el maltrato o el abuso del niño.

Las situaciones que dan origen a la visita suelen corresponder a problemáticas que ya están instaladas en los estudiantes y sus familias, lo que significa que la visita no se está utilizando como una estrategia de prevención social, sino de tratamiento, como respuesta tardía a un problema que está ocurriendo desde hace un tiempo y que ha generado efectos evidentes en el bienestar del estudiante y su proceso de aprendizaje, percibidos por los profesionales. De acuerdo con esto, podría mencionarse que la complejidad de los casos a intervenir mediante esta estrategia es alta, y frecuentemente requerirán de una combinación de estrategias implementadas con mucha mayor intensidad que las que se requerirían si se interviniera de manera temprana o a un nivel preventivo, siendo incluso necesaria la alianza de tipo intersectorial.

\section{Propósito de la visita domiciliaria}

Los participantes del estudio asociaron el propósito de la visita domiciliaria al proceso de conocer la situación sociofamiliar del estudiante, con la finalidad de conectarlos con redes que puedan ofrecerles el apoyo que requieran. Los entrevistados no la definen como una estrategia que en sí misma permita hacer tratamiento o generar cambios concretos en el ambiente familiar, que contribuyan a que el estudiante tenga un mejor proceso educativo. Esto se distancia de los planteamientos de Cook \& Sparks (2008), quienes le atribuyen a la visita el propósito de lograr el empoderamiento de la familia, como también concuerdan Reininger \& Santana (2015), que proponen un programa de visitas, hecho desde la escuela, orientadas a 
favorecer al involucramiento familiar colaborativo de los padres y apoderados, a través de un proceso de acompañamiento familiar.

Solo un entrevistado apostaría por un mayor potencial de la visita, aunque con limitaciones prácticas:

Sería interesante que la visita pudiera ser instrumento más propositivo, que uno pudiera ir no tan solo a mirar, porque acá trabajamos desde la urgencia; acá lo importante es mirar para poder tomar acciones ante ciertas situaciones que son complejas. Sería interesante poder tener el tiempo. Siento que la visita podría tener otro rol que sea más propositivo, hacer charlas al interior de la casa, trabajar por un tiempo más prolongado con las familias. Hacer un trabajo más personalizado, para que las familias puedan saber cómo funciona la red... Quizás hacer varias sesiones, hacerle un seguimiento a la familia. Pero eso por tiempo no se puede dar, y yo sé que las familias agradecerían bastante hacer un trabajo al interior de sus casas, estar ahí, hacer un trabajo más cercano y constante (E1).

Este tipo de propósito — centrado en el diagnóstico — podría explicar la irregular periodicidad con la que se aplican las visitas domiciliarias. Posiblemente también influyan en ello las dificultades en la coordinación de los tiempos profesionales, como se refleja en el siguiente extracto de entrevista:

Mm, por ejemplo, este mes que pasó hice cuatro, ahora en mayo hice una, puede que en un mes no haga ninguna y en otro haga seis; no es algo programado que yo tenga. Es muy difícil programar una visita, por ejemplo, acá tengo un calendario y tengo una persona a tal hora, pero resulta que quince minutos antes pasó otra situación, y eso impide un poco programarse. Pero, generalmente, al mes, haciendo un promedio son unas tres o cuatro visitas (E3).

Las visitas observadas tuvieron una duración diversa. El $68 \%$ duró menos de 40 minutos; el $28 \%$, entre 41 y 60 minutos, y solo el $4 \%$ duró más de 60 minutos. Si bien el tiempo de interacción producido entre el agente visitador y el usuario no garantiza la calidad de la visita, sí indica más posibilidades de formar un mejor vínculo y generar una conversación más profunda, de acuerdo con el objetivo para el cual esta haya sido efectuada.

\section{Actores de la visita domiciliaria: agente visitador y persona visitada}

En todos los casos observados en este estudio el agente visitador fue un profesional. A la vez, en el $100 \%$ de los casos estuvo presente el trabajador social con un papel protagónico. El $64 \%$ de las visitas observadas 
fueron realizadas individualmente, solo por el trabajador social, mientras que un $21 \%$ fueron realizadas por una dupla psicosocial: trabajador social y psicólogo. Solo en el $11 \%$ de los casos fueron realizadas con la compañía de un profesor.

Si bien son mínimos los casos en los cuales asisten profesores a la visita, cuando esto sucede es muy bien evaluado por el trabajador social en términos del compromiso del docente. Sin embargo, el papel que asumen no es claro durante la visita, como lo refleja el discurso del siguiente entrevistado:

Generalmente voy solo, aunque a veces hay profesores que son muy preocupados y jugados con los alumnos y también quieren acompañarnos. Pero, en la experiencia personal, cuando he ido con profesores solo se dedican a observar y más desde el ámbito pedagógico (E4).

Esta exigua participación de los profesores en las visitas es diferente de lo que sucede en otras experiencias internacionales, como la del modelo PTHV, implementada en varios estados de Estados Unidos. En esta experiencia no son los trabajadores sociales los que realizan las visitas, sino los mismos docentes, formados y recompensados para este fin (Sheldon \& Jung, 2018).

Con respecto a la persona visitada, en la mayoría de los casos quien interactuaba con el profesional era la madre del estudiante, con un $74 \%$ de las visitas observadas, seguido muy de lejos por tíos (8\%), hermanos (7\%), ambos padres (4\%) y otros (vecinos, abuelos) (7\%). De este modo, se identifica que desde el hogar son principalmente las mujeres que tienen el papel de madre quienes asumen la relación con la escuela, consistente con lo que señalan Hornby \& Lafaele (2011).

\section{Aspectos éticos de la visita domiciliara: presentación, información, coordinación de la visita}

Uno de los principales temas éticos se refiere al derecho de que las familias tengan la información sobre quién realiza la visita, su objetivo y el momento en el que esta se efectúa. En este estudio, la mayoría de los entrevistados revelaron que no acostumbran dar aviso a las familias sobre la visita a efectuar, como se aprecia en el siguiente extracto de entrevista:

O sea, la visita no se avisa. Nosotras llegamos de sorpresa a la casa, porque tampoco nosotras la tenemos programada una semana antes. O sea, si no es un caso tan grave de inasistencia, llamamos antes; si, por ejemplo, no contestan el teléfono, enviamos citación, y [si] no vienen, vamos a visita 
sin avisar que vamos a ir, porque si no... Pero no les avisamos, porque está el factor de que pueden no recibirnos si es que saben con antelación que vamos a ir, o pueden ir a otro lado (E5).

Las razones que sustentarían esta práctica serían disminuir la probabilidad de rechazo de la visita por parte de las familias (que no abran la puerta o que no estén), lo que podría reflejar que los entrevistados opinan que esta no es vista como una instancia grata o constructiva por parte de las familias. También señalan que otra de las razones es evitar que las familias construyan una realidad artificial, ocultando información relevante para la comprensión del caso, especialmente sensible en casos de vulneración de derechos de los niños.

Mira, la estrategia que he ocupado es no avisar visitas domiciliarias. A lo mejor, hay un cuestionamiento de otros colegas, pero en la práctica me he dado cuenta que conozco realmente a la familia, no me maquetean su realidad; así se conoce realmente a la familia. Y esa es una estrategia que yo he tomado, y me doy cuenta que ha servido (E4).

Consistentemente con lo arrojado por las entrevistas, en las observaciones realizadas se detectó que en la mayoría de los casos la visita no había sido coordinada con los padres y que, en consecuencia, los padres no estaban informados de su realización ni de sus objetivos. Esto se distancia de lo sugerido por Wasik \& Bryant (2001), que sugieren actuar siempre con honestidad en la visita domiciliaria, a no ser que la mentira del profesional pudiera proteger la integridad de una persona. En todo caso, "las circunstancias bajo las cuales mentir es justificable deben ser cuidadosamente consideradas y en casos relativamente extremos" (Wasik \& Bryant, 2001, p. 211).

Otro aspecto ético relevante es la solicitud de autorización a los padres, por parte del profesional, para compartir la información sociofamiliar recogida en la visita con los profesores $u$ otros miembros de la escuela. Si bien es habitual que durante las visitas emerja información privada y relevante con respecto a los niños, no parece ser un tema acordado con las familias el contar con el apoyo de los padres para compartir esa información, como se evidencia en esta entrevista:

La otra vez me pasó con el caso de un niño que la mamá tenía problemas de consumo de droga, y la hermana mayor cuidaba de este niño, pero esta hermana tenía dos niños bebés. Entonces les fui a hacer visita, y, claro, ellos vivían de allegados. Y explicarle toda esa situación a la profesora fue como "Yo no tenía conocimiento de eso". Entonces, igual te sirve para comentarle a los profesores en qué situaciones viven los niños (E8). 
Según Wasik \& Bryant (2001), la confidencialidad es una obligación ética del visitador del hogar, principio que está incluido en los códigos de ética de las profesiones de ayuda. Solo podría ser violada si el bienestar de las personas está en peligro. En este sentido, los autores afirman que comunicar y respetar la confidencialidad es un aspecto esencial en el proceso de construcción de una relación de confianza.

Por otra parte, de acuerdo con las observaciones realizadas, en un $89 \%$ de los casos el agente visitador no portaba ni credencial ni uniforme que le permitiera a la familia identificarlo en el momento de su arribo al hogar, lo que puede tener implicaciones que debiliten la percepción de seguridad del usuario, al no poder identificar con certeza quién es la persona que ingresará al hogar. Y, por otra parte, podría debilitar también la seguridad del profesional, el cual puede ser confundido o pasar desapercibido para la comunidad del sector.

\section{Percepción de la efectividad de la visita domiciliaria}

Los entrevistados aseveran que, en general, sí detectan cambios positivos en los estudiantes y sus familias tras la realización de una visita domiciliaria. En el caso del problema del ausentismo escolar, se percibe una mejora en el nivel de asistencia, aunque no es posible determinar si es por la conversación sostenida durante la visita o por el temor de ser denunciado en alguna instancia de protección de derechos del niño, como se puede ver en el siguiente extracto de entrevista:

Si me hablas de cambios, claro, se ve una implicación mayor a la que había, pero también uno tendría que decir qué motivó ese movimiento, si el temor, el miedo, el control social, o lo motivó un discurso disuasivo... A eso no llegamos. O sea, el efecto práctico era más que nada decir que si se mantenían las situaciones como estaban a ese momento, no iba a haber continuidad de matrícula o se iban a tomar medidas de protección, porque veíamos que los niños no estaban protegidos, niños muy de calle, etc. (E6).

Esta percepción sobre los efectos positivos de la visita en la asistencia a la escuela ha sido empíricamente comprobada por estudios internacionales, como la evaluación al modelo PHTV, que arrojó que las escuelas que implementan esta experiencia de manera sistemática disminuyen sus tasas de ausentismo crónico y aumentan los resultados en las pruebas de lenguaje y matemáticas (Sheldon \& Jung, 2018).

Sin embargo, los entrevistados enfatizan en que la visita por sí misma no es capaz de producir un cambio sostenible a largo plazo si no hay una alianza o complemento con otras acciones que desarrolle la escuela 
hacia el mismo objetivo de reducir la inasistencia, como lo manifiesta el siguiente entrevistado:

Inmediato sí, pero no sé si a largo plazo... Pero es una cosa de la escuela también, porque la escuela no registra los atrasos, no lo ven como parte de la formación educativa el que lleguen tarde. Entonces hay un problema de gestión en general como de la escuela que hay que ver, todavía no se ha encontrado la estrategia para registrar todos los atrasos, porque son como cien personas por día que llegan tarde (E7).

Otro de los cambios que se percibe positivamente por parte de los profesionales al efectuar las visitas es el desarrollo de una relación de más cercanía con el estudiante y sus familias, producto del conocimiento de la realidad familiar que permite la visita y la manifestación de preocupación de la escuela hacia el niño.

\section{Dificultades y ventajas de la visita domiciliaria en el contexto escolar}

Los participantes del estudio identifican tanto ventajas como dificultades en la realización de visitas domiciliarias en el contexto escolar.

\section{Dificultades o limitaciones de la visita domiciliaria}

Son dos las principales dificultades o limitaciones que los entrevistados encontraron en la realización de visitas domiciliarias. La primera de ellas es la desconfianza que tienen las familias acerca de las intenciones del agente visitador, las cuales interpretarían como vinculadas con el control y la denuncia o con la comunicación de noticias negativas sobre el estudiante, tal como se aprecia en el siguiente extracto de entrevista:

Las dificultades yo creo que es la desconfianza por parte de la familia para la realización de la visita domiciliaria; yo creo que la mayoría de las familias ve y cree que uno visita su domicilio con la finalidad de hacer una denuncia. Porque la OPD es como los carabineros para los delincuentes. Ellos desconfían mucho de la visita domiciliaria y de la función de la trabajadora social de la escuela. Tienes que hacer un trabajo de por medio para que te vean como una amiga y no como una enemiga, como que vean que tú en realidad vas a su casa para ver el estado de la casa y entregar herramientas o ayuda de lo que requieran en el momento, y no para ver qué es lo que les falta o cuáles son las deficiencias como padres (E2).

Esa desconfianza actúa como una barrera importante para la relación con el profesional, la cual produce distancia entre el agente y el usuario y 
genera, como posibles consecuencias, que no abran la puerta del hogar o que no permitan el ingreso del profesional. De hecho, se detectó que el profesional no pudo ingresar al hogar de la familia del estudiante en casi la mitad de las visitas, teniendo que efectuar la conversación en la vereda o en el pasillo del edificio. El realizar la visita fuera del hogar es indicativo de desconfianza y baja disponibilidad del usuario hacia el profesional, lo que merma la posibilidad de desarrollar un vínculo, disminuye el tiempo de la visita por la incomodidad y baja la calidad de la interacción, de modo que se afectan los resultados de la estrategia.

La segunda de las limitaciones sería la incapacidad de la visita domiciliaria - así como es implementada por ellos- para poder producir cambios en familias que tienen problemáticas muy complejas y que demuestran poco compromiso con el proceso educativo del estudiante. De este modo, la coordinación con otras instituciones de apoyo, como las del ámbito de la salud, y el trabajo intersectorial serían fundamentales para hacer frente a ese tipo de situaciones, tal como lo señala este entrevistado:

Yo creo que ahí hay una cuestión súper importante de coordinación, porque yo no sé cuáles son las visitas domiciliarias que está teniendo una familia que yo atiendo de un equipo de salud o de otros actores de la institucionalidad. Por lo tanto, cómo me coordino para ver si estoy haciendo lo mismo. Por eso te digo, a mí me cuesta ponerle las fichas a una intervención en terreno a partir de una visita si no hay una cosa intersectorial, que esté más coordinada con otras instituciones, donde lo mío sea un complemento y que no vaya a hacer la pega del Cesfam o viceversa (E6).

Concordando con este tema planteado por los entrevistados, el trabajo en equipo y colaborativo es sugerido por Cook \& Sparks (2008), quienes valoran que los miembros del equipo adopten un modelo de trabajo transdisciplinario, tanto para diseñar como para implementar la intervención a través de las visitas. Eso sí, los autores advierten que hay que cuidar que las familias no se estresen ni frustren por la presencia de demasiadas personas en el hogar ni por la necesidad de coordinar los servicios (Cook \& Sparks, 2008).

\section{Fortalezas y ventajas de la visita domiciliaria}

La oportunidad para forjar un vínculo más profundo con las familias de los niños sería la principal fortaleza de la visita. Este vínculo permite conocer de manera más integral y fidedigna la situación del estudiante, la cual en algunos casos está afectada por una variedad de problemáticas sociales que influyen en su desarrollo educativo, como lo manifiesta el siguiente extracto de entrevista: 
Algo positivo desde mi área es poder conocer un poco más la realidad del niño: sabes la problemática que tiene, sabes cómo vive, sabes que esas cosas influyen en su comportamiento... Entonces explicar también a los profesores que a veces no saben toda la realidad del niño (E8).

Con respecto a esta fortaleza, hay autores que concuerdan en que la relación entre los padres y el visitador del hogar sería la esencia de la visita domiciliaria (Cook \& Sparks, 2008; Klass, 2008), una relación orientada a maximizar la posibilidad de promover el desarrollo de los padres y, con ello, el de los niños. Esta es una relación dinámica, que varía en su naturaleza y efectos como resultado de las características personales de los padres y el visitador, y de las dinámicas del ambiente.

En pocos casos los entrevistados señalaron qué actores de la comunidad educativa son los que tienen que comprender la situación sociofamiliar del niño, no siendo claros en su discurso si es una información que solo reviste interés para el trabajador social o también para el profesor, de modo que pueda contextualizar mejor su labor educativa, tal como lo esboza el entrevistado número 8 (E8). La posibilidad de compartir esa información con los profesores es una estrategia valiosa para poder comprender la situación del niño en la escuela y estructurar un proceso educativo especializado.

Otra de las ventajas es que, en un contexto escolar masivo con un grupo de estudiantes tan numeroso, la visita domiciliaria constituye una ocasión individual, de consideración y deferencia hacia las familias, que no se obtiene en otras instancias escolares, lo cual produce un sentimiento de inclusión que genera una disposición positiva hacia la escuela por parte de la familia, como se aprecia en el siguiente discurso:

Yo creo que lo bueno por ese lado es que puedes hacer como un lazo con el apoderado, conocerlo un poco más y, como te dije y como he repetido todo el rato, que vean la preocupación del colegio hacia ellos. Porque, de verdad, acá, bueno, generalmente, como es un colegio grande, uno no se siente tan considerado, porque son muchos alumnos, porque son muchos apoderados. Entonces quizás eso también los hace sentirse parte de algo, de una comunidad... Entonces yo creo que es súper importante para integrarlos y para que también participen en los temas del colegio (E5).

Otro aspecto que facilita la aplicación de la visita es el contar con un director de escuela que valore y apoye la aplicación de esta estrategia, reconociéndola como una herramienta apropiada en el contexto escolar y aceptando que los profesionales ocupen parte de su tiempo profesional en ella. 


\section{Discusión y conclusiones}

En el tema de la participación de las familias en la educación abundan los estudios que evidencian su impacto positivo, aunque son pocos los que proponen y profundizan en iniciativas para potenciar dicha relación en el sistema educativo (Weiss, Bouffard, Bridglall \& Gordon, 2009). Este artículo contribuye a aportar en este ámbito, con base en un estudio realizado en escuelas en contextos vulnerables. El estudio en estos contextos es valioso si se considera que las capacidades de las familias para llevar a cabo su cometido educativo no están equitativamente distribuidas, puesto que la pobreza, el nivel educativo de los padres y su capital social influyen en el desarrollo y el desempeño escolar de niños y adolescentes (Razeto, 2016a). Los estudios para fomentar la participación de las familias son necesarios, por cuanto esta propicia una mayor equidad al atenuar la influencia negativa del contexto social desfavorable (Valdés \& Urías, 2011). Por lo tanto, la visita domiciliaria se presenta como una estrategia muy práctica y con un potencial interesante desde el punto de vista de su aporte a la implicación de las familias que presentan más barreras para participar en la educación de sus niños a través de las actividades organizadas en la escuela, producto de su exclusión social.

A diferencia de lo postulado por Franklin \& Lagana-Riordan (2009) y Paulsell et al. (2010), en este estudio se encontró que las visitas estaban principalmente enfocadas en remediar el problema del ausentismo escolar, una vez que este ya se presentaba de manera evidente en la escuela. Esta situación indica que posiblemente el uso que se le está dando a las visitas a nivel nacional es limitado y restringido, sin apreciar sus ventajas como estrategia de prevención social y tratamiento (Aracena et al., 2011). Esto contrasta con la necesidad de desarrollar acciones educativas especialmente dirigidas a los padres, mostrándoles las posibles formas en que pueden participar en la educación de sus hijos. De hecho, esta necesidad ha sido reconocida por las mismas familias, como lo plantea un estudio realizado por Valdés \& Urías (2011).

Esta tendencia nacional a restringir el uso de la visita como herramienta de evaluación o diagnostica concuerda con lo encontrado en el estudio realizado por Chamorro \& Razeto (2016) en el contexto de las prácticas de un grupo de estudiantes de Trabajo Social. No se trata de desconocer el valor que tiene la comprensión de las condiciones reales de las familias de los alumnos, cuestión fundamental según Castro \& Regattieri (2012), sino de valorar que esa información puede ser utilizada por las escuelas para delimitar su responsabilidad y planear los apoyos para aquellas familias menos implicadas en la educación (Castro \& Regattieri, 2012). 
Otros de los resultados interesantes del estudio es el relacionado con el tema ético de las visitas, puesto que se encontró que existía una tendencia a no informar de su realización a las familias, por la creencia de que estas podrían rechazarla o modificar su realidad. Esto sería contrario a la sinceridad con la que tiene que actuar el agente visitador, recomendada por Allen \& Tracy (2004). En el ámbito de la salud y a nivel nacional, el Ministerio de Salud (2009) también recomienda que las visitas domiciliarias se realicen con el acuerdo explícito de la familia. Por lo tanto, es posible plantear que esta práctica de no coordinar de forma transparente con la familia perjudica la calidad de la interacción y los cambios que son posibles de alcanzar con el uso de esta estrategia. Otra práctica que podría vulnerar la ética es la transmisión de información sobre la situación de los niños a los docentes o centro educativo sin considerar el permiso explícito de sus padres o tutores.

Con respecto a los agentes visitadores, en todos los casos se observó la presencia de los trabajadores sociales como protagonistas de las visitas domiciliaras. Esto concuerda con el valor histórico y estratégico que tiene la visita domiciliaria como estrategia de intervención para el trabajo social (Tonon, 2005). No obstante, el hecho de que las visitas sean realizadas por trabajadores sociales de nivel profesional no garantiza la eficacia de la estrategia, puesto que una de las variables relevantes para ello es el nivel de capacitación de los que hacen la visita domiciliaria, independientemente de si estos son o no profesionales (Tubach et al., 2012). Por otro lado, la literatura documenta la participación de otros profesionales en las visitas, además de los trabajadores sociales, como es el caso del programa Family Engagement Partnership, evaluado en el año 2015 por la Universidad de John Hopkins, en las que participan activamente los profesores o educadores (Center for Social Organization of Schools, John Hopkins University, 2015).

Finalmente, es posible destacar que las ventajas de la visita y la efectividad percibida por los entrevistados, si bien son consistentes con numerosa literatura que comprueba científicamente los efectos positivos de la visita en otros ámbitos (Ascencio-Huertas, Rangel \& Allende, 2013; Avellar \& Supplee, 2013; Brugerolles, Dois \& Mena, 2008; Cruz \& Marthe, 2010; Gogia \& Sachdev, 2010; Issel, Forrestal, Slaughter, Wiencrot \& Handler, 2011), tienden a restringirse solo a dos aspectos: la calidad del vínculo generado y el sentimiento de reconocimiento e inclusión que les genera en la escuela. No se reconoce el positivo potencial que tiene el hogar, no solo para observar sino para modelar, enseñar e influir en la interacción de sus miembros, reconociendo que ninguna intervención será efectiva si no entra en las rutinas diarias de la familia (Bernheimer \& Weisner, 2007). En este proceso de influencia, es necesario adoptar un enfoque de trabajo colaborador, que no enjuicie, sino que valide las prácticas positivas que las familias 
tienen en relación con la educación de sus hijos, desarrollando acuerdos para cambiar o mejorar las que sean pertinentes.

Este estudio deja abiertas varias interrogantes que podrían ser objeto de nuevas investigaciones, tales como el significado que le atribuyen otros miembros de la comunidad escolar a las visitas, como los profesores y directores. También se podría explorar qué es lo que ha cambiado tras ellas, que se pueda explicar, por ejemplo, la disminución del ausentismo. Otra interrogante podría ser evaluar cuáles han sido los posibles cambios en los resultados de aprendizaje tras la visita, estudio que exigiría un planteamiento más longitudinal. Finalmente, en un objeto de estudio más amplio, sería interesante explorar con más profundidad la práctica profesional cotidiana de los trabajadores sociales ubicados en escuelas, analizando sus cuestiones éticas y reconociendo otras técnicas con las que desarrollan su labor.

La principal limitación del estudio fue el tamaño de la muestra de entrevistados y las posibles interferencias del investigador en el proceso de observación realizado en terreno.

\section{Sobre la autora}

Alicia Cristina Razeto-Pavez es académica de la Escuela de Trabajo Social de la Facultad de Ciencias Sociales de la Pontificia Universidad Católica de Chile, Chile. Doctora en Ciencias de la Educación de la Pontificia Universidad Católica de Chile, Chile. Tiene estudios de posdoctorado en el Grupo de Educación y Trabajo de la Universidad Autónoma de Barcelona, España.

\section{Referencias}

Allen. S. \& Tracy, E. (2004). Revitalizing the role of home visiting by school social workers. Children \& Schools, 26(4), 197-207. doi: https://doi.org/10.1093/ $\mathrm{cs} / 26.4 .197$

Aracena, M. et al. (2011). Evaluación de la efectividad de programas de visitas domiciliarias para madres adolescentes y sus hijos/as. Revista Médica de Chile, 139(1), 60-65. doi: http://dx.doi.org/10.4067/S0034-98872011000100008

Arevalo, M. \& Santana, A. (2015). Percepción de los resultados de la intervención social implementada por duplas psicosociales en escuelas municipales. Implicancias en la vinculación familia-escuela. En M. Saracostti \& C. Villalobos (Eds.), Familia-escuela-comunidad III: implementando el modelo de intervención biopsicosocial (pp. 93-120) Santiago: Universitaria.

Ascencio-Huertas, L., Rangel-Domínguez, N. \& Allende, S. (2013). Proceso de atención psicológica en el programa de atención y cuidados en oncología del Instituto Nacional de Cancerología. Psicooncología, 10(2-39), 393-406. Recuperado de http://revistas.ucm.es/index.php/PSIC/article/view/43457

Avellar, S. \& Supplee, L. (2013). Effectiveness of home visiting in improving child health and reducing child maltreatment. Pediatrics, 132(2), 90-99. 
Baquedano-López, P., Alexander, R. A. \& Hernández, S. J. (2013). Equity issues in parental and community involvement in schools: What teacher educators need to know. Review of Research in Education, 37(1), 149-182. doi: http:// dx.doi.org/10.3102/0091732X12459718

Bernheimer, L. \& Weisner, T. (2007). "Let me just tell you what I do all day...": The family story at the center of intervention research and practice. Infants \& Young Children, 20(3), 192-201. Recuperado de https://depts.washington. edu/isei/iyc/20.3_bernheimer.pdf

Blanco, R. \& Umayahara, M. (2004). Participación de las familias en la educación infantil latinoamericana. Recuperado de http://unesdoc.unesco.org/ images/0013/001390/139030s.pdf 2004

Boberiene, L. V. (2013). Can policy facilitate human capital development? The critical role of student and family engagement in schools. American Journal of Orthopsychiatry, 83(2), 346-351. doi: https://doi.org/10.1111/ajop.12041

Braun, V. \& Clarke, V. (2006). Using thematic analysis in psychology. Qualitative Research in Psychology, 3(2), 77-101. Recuperado de http://dx.doi. org/10.1191/1478088706qp063oa

Brugerolles, M., Dois, A. \& Mena, C. (2008). Beneficio de la visita domiciliaria para los adultos mayores. Horizonte de Enfermería, 1(2), 55-64. doi: 10.7764/Horiz_Enferm.19.2.55

Castro, J. \& Regattieri, M. (2012). Interacción escuela-familia. Insumos para las prácticas escolares. Brasilia: Unesco \& Ministerio de Educación. Recuperado de http://unesdoc.unesco.org/images/0021/002163/216310s.pdf

Center for Social Organization of Schools, John Hopkins University. (2015). The Family Engagement Partnership. Student Outcome Evaluation. Recuperado de http://www.pthvp.org/wp-content/uploads/2016/09/JHU-STUDY_FINALREPORT.pdf

Chamorro, P. \& Razeto, A. (2016). Visita domiciliaria en trabajo social ¿cómo la valoran y utilizan un grupo de estudiantes de la profesión? Revista de Trabajo Social, 89(1), 15-28. Recuperado de http://rts.alerta.cl/index.php/ rts/article/view/29

Cook, R. \& Sparks, S. (2008). The Art and Practice of Home Visiting. Maryland, EE. UU.: Brookes.

Cruz, M. \& Marthe, M. (2010). A visita domiciliária na estratégia de saúde da família: Conhecendo as percepções das famílias. Saúde e Sociedade, 19(3), 605-613. Recuperado de http://dx.doi.org/10.1590/S010412902010000300012

Dikkers, A. G. (2013). Family connections: Building connections among home, school, and community. Childhood Education, 89(2), 115-116. doi: 10.1080/00094056.2013.774247

Epstein, J. (2011). School, Family and Community Partnerships. California: Westview.

Franklin, C. \& Lagana-Riordan, C. (2009) School-based services. En S. Allen \& E. Tracy (Eds.), Delivering Home-based Services: A Social Work Perspective (pp. 111-133). Nueva York: Columbia University Press.

Garcia-Reid, P., Peterson, C. H. \& Reid, R. J. (2013). Parent and teacher support among latino immigrant youth: Effects on school engagement and 
school trouble avoidance. Education and Urban Society, 47(3), 328-343. doi: $10.1177 / 0013124513495278$

Gil, J. (2009). Hábitos y actitudes de las familias hacia la lectura y competencias básicas del alumnado. Revista de Educación, 5(350), 301-322.

Gogia, S. \& Sachdeva, H. (2010). Home visits by community health workers to prevent neonatal deaths in developing countries: A systematic review. Bull World Health Organ, 88(9), 658-666. doi: 10.2471/BLT.09.069369

Hoover-Dempsey, K., Walker, J., Sandler, H., Whetsel, D., Green, C., Wilkins, A. \& Closson, K. (2006). Why do parents become involved? Research findings and implications. The Elementary School Journal, 106(2), 105-130.

Hornby, G. \& Lafaele, R. (2011). Barriers to parental involvement in education: An explanatory model. Educational Review, 63(1), 37-52. doi: 10.1080/00131911.2010.488049

Issel, L., Forrestal, S., Slaughter, J., Wiencrot, A. \& Handler, A. (2011). A review of prenatal home-visiting effectiveness for improving birth outcomes. Journal of Obstetric, Gynecologic and Neonatal Nursing, 40(2), 157-165. doi: https://doi.org/10.1111/j.1552-6909.2011.01219.x

Kearney, C. (2016). Managing School Absenteeism at Multiples Tiers. An Evidence-Based. Nueva York: Oxford University Press.

Kim, J. \& Streeter, C. (2013). Increasing school attendance: Effective strategies and interventions. En C. Franklin, M. Harris \& P. Allen-Meares, The School Services Sourcebook: A Guide for School-Based Professionals (pp. 395404). Nueva York: Oxford University Press.

Klass, C. (2008). The Home Visitor's Guidebook. Promoting Optimal Parent \& Child Development. Baltimore: Brookes.

Lauchlan, F. (2003). Responding to chronic non-attendance: A review of intervention approaches. Educational Psychology in Practice, 19(2), 133-146. doi: 10.1080/02667360303236

Ministerio de Salud. (2009). Visita domiciliaria integral para el desarrollo biopsicosocial de la infancia. Orientaciones técnicas. Recuperado de http://www. crececontigo.gob.cl/wp-content/uploads/2015/11/Visita-Domiciliaria-Inte gral-para-el-Desarrollo-Biopsicosocial-de-la-Infancia-2009.pdf

Paulsell, D., Boller, K., Hallgren, K. \& Mraz, A. (2010). Assessing home visit quality: Dosage, content, and relationships. Zero to Three (j), 30(6), 16-21.

Pellegrini, D. (2007). School non-attendance: Definitions, meanings, responses, interventions. Educational Psychology in Practice, 23(1), 63-77. doi: 10.1080 /02667360601154691

Razeto, A. (2016a). El involucramiento de las familias en la educación de los niños. Cuatro reflexiones para fortalecer la relación entre familias y escuelas. Páginas de Educación, 19(2). Recuperado de https://revistas.ucu.edu.uy/ index.php/paginasdeeducacion/article/view/1298

Razeto, A. (2016b). Estrategias para promover la participación de los padres en la educación de sus hijos: el potencial de la visita domiciliaria. Estudios Pedagógicos, 42(2), 449-462. doi: http://dx.doi.org/10.4067/S071807052016000200026

Reid, K. (2013). An Essential Guide to Improving Attendance in your School. Nueva York: Routledge. 
Reininger, T. \& Santana, A. (2015). Fortaleciendo vínculos: un programa de Ciclo de Visitas Domiciliarias para familias de primer año de educación básica. En M. Saracostti \& C. Villalobos (Eds.), Familia-escuela-comunidad III: implementando el modelo de intervención biopsicosocial (pp. 211-234). Santiago: Universitaria.

School Social Work Association of America (SSWAA). (2019). The roles of a school social workers. Recuperado de https://aab82939-3e7b-497d8f30-a85373757e29.filesusr.com/ugd/426a18_efad06f06f4f4329985d 5d1961603352.pdf

Sheldon, S. \& Jung, S. (2018). Student Outcomes and Parent Teacher Home Visits. Center on School, Family, \& Community Partnerships, Johns Hopkins University. Recuperado de http://www.pthvp.org/wp-content/uploads/20 18/12/181130-StudentOutcomesandPTHVReportFINAL.pdf

Tonon, G. (2005). Las técnicas de actuación profesional del trabajo social. Buenos Aires: Espacio.

Tubach, F. et al. (2012). A home-visiting intervention targeting determinants of infant mental health: The study protocol for the CAPEDP randomized controlled trial in France. BMC Public Health, 12, 648. doi: 10.1186/14712458-12-648

Valdés, A. \& Urías, M. (2011). Creencias de padres y madres acerca de la participación en la educación de sus hijos. Perfiles Educativos, 33(134), 99-114. Recuperado de http://www.scielo.org.mx/scielo.php?script=sci_arttext\&p id $=$ S0185-26982011000400007

Vera, J., González, C. \& Hernández, S. (2014). Familia y logro escolar en matemáticas del primer ciclo escolar de educación primaria en Sonora, México. Estudios Pedagógicos, 40(1), 281-292.

Villalobos, C. (2015). El rol de la intervención social en el sistema escolar chileno. Una reflexión del aporte del Trabajo Social a una educación integral e inclusiva en el marco de la Reforma. Revista Debates, 56-62. Recuperado de https://www.academia.edu/15998520/El_rol_de_la_intervenci\%C3\%B3n_ social_en_el_sistema_escolar_chileno._Una_reflexi\%C3\%B3n_del_apor te_del_Trabajo_Social_a_una_educaci\%C3\%B3n_integral_e_inclusiva_en_ el_marco_de_la_Reforma

Wasik, B. \& Bryant. D. (2001). Home Visiting. Procedures For Helping Families. Thousand Oaks, CA: Sage.

Weiss, H., Bouffard, S., Bridglall, B. \& Gordon, E. (2009). Reframing Family Involvement in Education: Supporting Families to Support Educational Equity. Research Review 5. Recuperado de https://files.eric.ed.gov/fulltext/ ED523994.pdf

Zamorano, L. (1935). El servicio social escolar (tesis de pregrado). Pontificia Universidad Católica de Chile, Santiago, Chile. 